\title{
CYTOLOGICAL AND ISOENZYME ANALYSIS OF THE BUCAY AND QUEVEDO CYTOTYPES OF THE ONCHOCERCIASIS VECTOR SIMULIUM EXIGUUM (DIPTERA: SIMULIIDAE) IN ECUADOR
}

\author{
M. Charalambous; P. D. ReADY; A. J. SHELLEY; M. ARZUBE* \& C. A. LOWRY
}

\begin{abstract}
Department of Entomology ${ }^{+}$, The Natural History Museum, Cromwell Road, London SW7 5BD, U. K. *Instituto de Higiene y Medicina Tropical 'Leopoldo Izquieta Perez', Guayaquil, Ecuador
\end{abstract}

Four cytotypes of Simulium exiguum occur in Ecuador, where this morphospecies is the primary vector of onchocerciasis. In this paper, we give the first full description of the banding pattern of the larval polytene chromosomes of the Quevedo cytotype and assess its degree of genetic separation from the Bucay cytotype. Both cytotypes differ from the chromosomal standard sequence (of the Cayapa cytotype) by the fixed inversions IIL-5 and IIL-6. The Quevedo cytotype additionally differs from the standard and Bucay cytotypes by possessing a differentiated $X$ chromosome, which is indicated by the inversion IIS-A. As the degree of reproductive isolation between the Bucay and Quevedo cytotypes has not yet been established, they must be regarded as intraspecific variants of the same species. In fact, isoenzyme characterizations showed that the Bucay and Quevedo cytotypes are differentiated only to the extent expected of incipient species or geographical populations. Moreover, the sibling species status previously given to the Bucay cytotype needs be reassessed, there being inadequate analysis from areas in Ecuador where Bucay occurs in sympatry with the standard Cayapa cytotype. No isoenzyme electromorphs were discovered that identified all or most adult females of any one (cytotypepure) collection.

Key words: Blackfly - Simulium exiguum - Onchocerca volvulus - species complex - cytotaxonomy isoenzymes - Ecuador

Simulium exiguum sensu lato is widespread in Latin America, occurring in Argentina, Bolivia, Brazil, Colombia, Ecuador, Peru and Venezuela (Shelley et al., 1989). It acts as the primary vector of Onchocerca volvulus in the foci of onchocerciasis found in the northwestern Province of Esmeraldas, Ecuador (Shelley \& Arzube, 1985; Shelley et al., 1985; Shelley, 1988; Shelley et al., 1989; Shelley et al., 1990) and is also implicated as a vector of this disease in Colombia and in Venezuela (Duke, 1970; Tidwell et al., 1980). Regional differences in the biology of $S$. exiguum, and in its susceptibility to infection by $O$. volvulus, led

This work was supported by The Natural History Museum, U.K.; Instituto de Higiene y Medicina Tropical, Ecuador; and the World Health Organization.

M. Charalambous is supported by a Senior Research Fellowship at The Natural History Museum.

${ }^{+}$World llealth Organization Collaborating Centre for the study of Simuliidae and Phlebotominae in relation to Onchocerciasis and Leishmaniasis.

Received 21 July 1992.

Accepted 21 September 1992.
Shelley \& Arzube (1985) to suggest that the morphospecies $S$. exiguum is a complex of sibling species. Four morphologically-indistinguishable cytotypes (named after primary collection sites) of $S$. exiguum are currently recognized in Ecuador (Procunier et al., 1985; Procunier, 1989). The epidemiological significance of these cytotypes is the subject of a multidisciplinary research programme in Ecuador, involving cytogenetic and biochemical analyses, some results of which are presented in this paper.

The two cytotypes recorded from the major focus of onchocerciasis in Esmeraldas Province (Fig. 1) give every indication of being good biological species: the Aguarico cytotype differs from the chromosomal standard banding pattern sequence (of the Cayapa cytotype) by two fixed inversions in the long arm of chromosome two (IIL), as well as by having distinct sex chromosomes and several autosomal polymorphisms; and, moreover, no heterozygotes for the interspecific IIL inversions have been recorded where the cytotypes are 


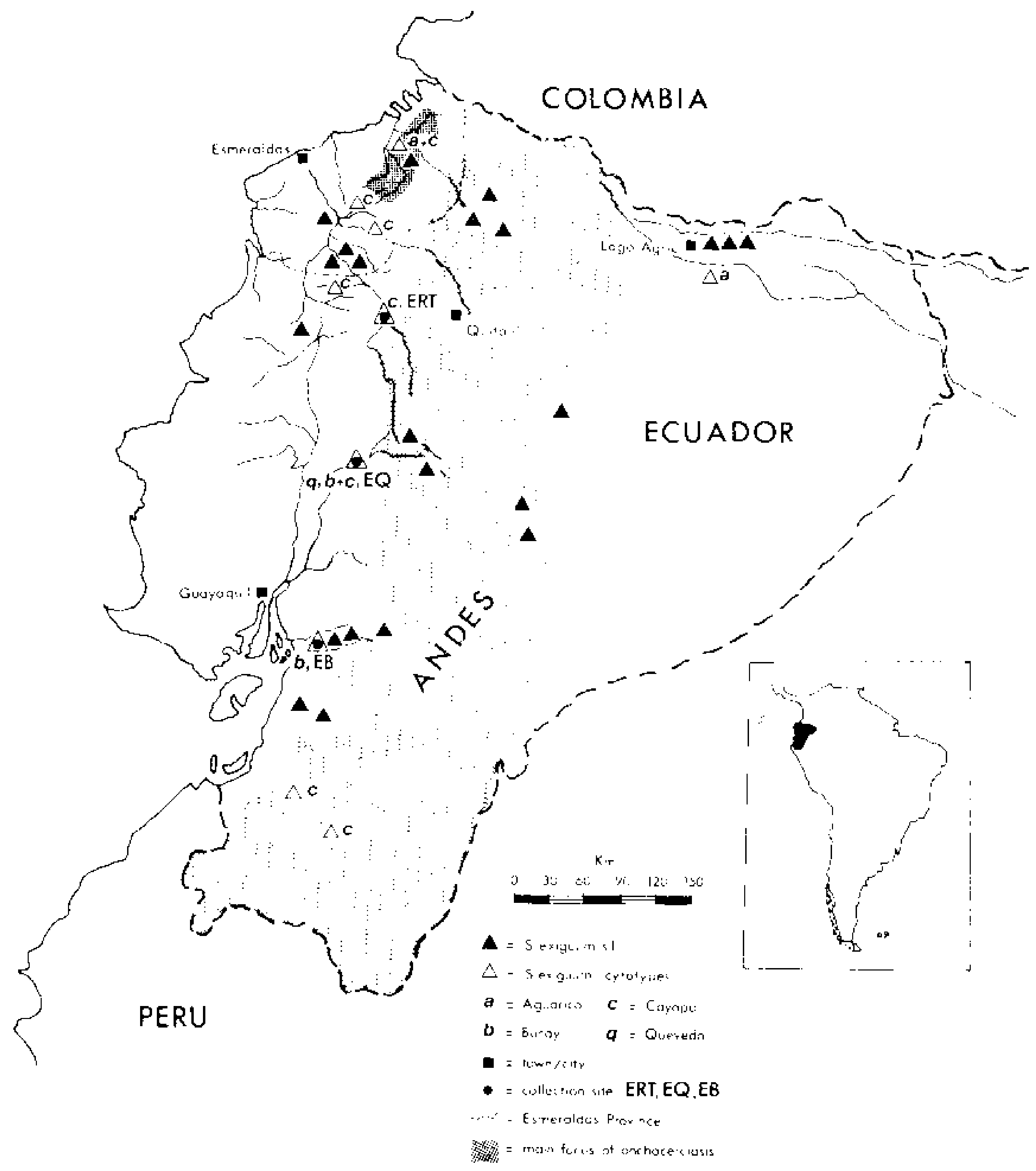

Fig. I: distribution of Simuliun exigurm in Ecuador. Closed triangles indicate sites where S. exiguum morphospecies has been found. Open triangles indicate sites where populations have been examined cytologically. Closed circles indicate the collection sites of the present study.

sympatric, although sample sizes are small (see Discussion) (Procunier et al., 1985). The Bucay cytotype also differs from the Cayapa cytotype by two fixed inversions on IIL (different from those of the Aguarico cytotype), but its specific status is less clear because it has been examined in detail from just one population far to the south of the onchocerciasis foci (Procunier et al., 1985; site EB in Fig. 1). The fourth cytotype has not been properly described, but was reported to be similar to the Bucay cytotype, from which it showed differences in the sex chromosomes (Procunier, 1989); the "type locality" of this Quevedo cytotype lies between Bucay and the foci of onchocerciasis (site EQ in Fig. 1).

In this paper, we report on the genetic relationship between the Bucay and Quevedo cytotypes using cytological and isoenzyme methods of characterization. A precise cytological description of the Quevedo cytotype is presented for the first time. All published findings have been based on the study of polytene chromosomes of larval salivary glands because, unfortunately, polyteny is not well developed in the nuclei found in adult tissues of $S$. exiguum (Procunier, 1989). This inability to characterize adults of the different cytotypes prompted us to start isoenzyme analyses, with the aim of finding diagnostic characters for adults as well as to identify polymorphic loci that will permit analysis of interbreeding between cytotypes and different populations.

\section{MATERIALS AND METHODS}

Collection sites - A number of collections of $S$. exiguum have been made since 1984 at three localities in Ecuador (sites EB, EQ, ERT; 
TABLE I

Summary of cytological results

\begin{tabular}{|c|c|c|c|c|c|c|c|c|}
\hline \multicolumn{3}{|c|}{ Collection } & \multirow[t]{2}{*}{ Date } & \multicolumn{2}{|c|}{ No. Identified } & \multicolumn{2}{|c|}{ Inversions } & \multirow[t]{2}{*}{ Cytotype } \\
\hline No. & Site & Rio/River & & Total & Males & Fixed & Sex & \\
\hline 1 & EB & Bucay & $19.9 .84^{a}$ & 17 & $?$ & IIL-5 + 6 & - & Bucay \\
\hline 2 & & & 24.7 .89 & 31 & 12 & IIL $-5+6$ & - & Bucay \\
\hline 3 & & & 11.9.91 & 30 & 12 & IIL $-5+6$ & - & Bucsy \\
\hline 4 & $\mathrm{EQ}$ & Pilaló & $8.6 .84 b$ & 1 & 0 & \multicolumn{2}{|c|}{ standard $c$} & Cayapa \\
\hline 4 & & & $8.6 .84^{b}$ & 10 & 3 & IIL-5 + 6 & - & Bucay \\
\hline 5 & & & 18.7 .89 & 13 & 6 & IIL-5 + 6 & IIS-A ${ }^{d}$ & Quevedo \\
\hline 6 & & & 24.9 .91 & 21 & 7 & IIL-5 + 6 & IIS-A $d$ & Quevedu \\
\hline 7 & ERT & Tanti & 14.3 .90 & 5 & 2 & \multicolumn{2}{|c|}{ standard $^{c}$} & Cayapa \\
\hline
\end{tabular}

$a$ : data from Procunier et al. (1985); $b$ : chromosome preparations in BM(NH) collection; $c$ : standard sequence of the Cayapa cytotype; $d$ : X-linked inversion.

see Fig. 1). Table I contains data from all available cytological analyses performed since 1984 , in order to present a complete picture of the cytological structure of populations at these localities.

Three collections, numbers 1-3, were made on the Rio Bucay (site EB) near the waterworks in Naranjal (latitude/longtude co-ordinates: $2^{\circ} 41^{\prime} \mathrm{S} / 79^{\circ} 36^{\prime} \mathrm{W}, 80 \mathrm{~m}$ altitude). Three collections, numbers 4-6, were made on the Río Pilaló (locally known as Río San Pablo) (site EQ), $2 \mathrm{~km}$ from La Mana on the Latacunga road $\left(0^{\circ} 56^{\prime} \mathrm{S} / 79^{\circ} 13^{\prime} \mathrm{W}, 260 \mathrm{~m}\right.$ altitude). One collection (number 7) has been made on the Rio Tanti (site ERT), $10 \mathrm{~km}$ from Santo Domingo de los Colorados on the Alluriquín road $\left(0^{\circ} 18^{\prime} \mathrm{S} / 79^{\circ} 5^{\prime} \mathrm{W}, 640 \mathrm{~m}\right.$ altitude).

The dates of collection are given in Table I. Collections 1 and 4 were made by M. Arzube, W. J. Procunier \& A. J. Shelley; collections 2 and 5 by M. Arzube \& A. J. Shelley; collections 3 and 6 by M. Arzube \& M. Charalambous; and collection 7 by J. Sawyer.

Old collections - The cytological data of collection 1 are from Procunier et al. (1985). The data from collection 4 are that present in the chromosome slide collection in The Natural History Museum (BM(NH)) (see Table I).

New collections $-S$. exiguum larvae and adults where collected from Ecuador between 1989 and 1991 (collections 2, 3, 5, 6 and 7). For each collection, larvae were placed into freshly-made cold Carnoy's fixative (75\% absolute ethanol: $25 \%$ glacial acetic acid) on site. The fixative was changed within a few hours, and larvae put into a refrigerator within three weeks of collection. Some pupae were collected and cultured individually until adult emergence, and the resulting link-reared adults were frozen in liquid nitrogen for biochemical studies. However, most adults used for isoenzyme work were females landing on man baits. These were stored in liquid nitrogen (Ecuador) and then in a $-70^{\circ} \mathrm{C}$ freezer (London).

Morphological identification - Larvae for cytotaxonomic determination were identified using criteria in Shelley et al. (1989). Adults, to be used in isoenzyme analysis, were superficially identified as $S$. exiguum in the field by their general coloration, the absence of scutal patterning and the predominantly yellow legs (Shelley et al., 1989). These identifications were later confirmed in the laboratory by studying the slide-mounted genitalia (particularly the paraproct) because of the possibility of finding the closely-related $S$. gonzalezi at the collection sites. Larvac, pupae, and link-reared adults of $S$. exiguum have been deposited in the collections of the $\mathrm{BM}(\mathrm{NH})$ and the Instituto de Higiene y Medicina Tropical, Ecuador.

Cytology - Polytene chromosome preparations were made using a modification of the Feulgen method (Dunbar, 1972). Penultimate instar larvae (with white pupal gill histoblasts) were split open ventrally, in fresh Carnoy's fixative, to expose the silk glands. Larvae were then placed in distilled water for $1 \mathrm{hr}$ and subsequently treated for $1 \mathrm{hr}$ in $5 \mathrm{~N} \mathrm{HCl}$ at room temperature. Following hydrolysis, larvae were rinsed in distilled water twice $(2 \mathrm{~min}$ each time) and placed in Feulgen for 0.5-2 hr. Feulgen was replaced with $\mathrm{SO}_{2}$ water $(\mathrm{lg}$ 
potassium metabisulphite in $200 \mathrm{ml}$ distilled water $+10 \mathrm{ml} 1 \mathrm{~N} \mathrm{HCl}$ ) for 10 minutes and, finally, cold tap water.

The silk glands were removed onto a slide, using mounted needles, and counterstained in lacto-propionic orcein for $5-20 \mathrm{~min}$ (depending on the amount of initial staining), which was then blotted off. Silk glands were mounted in a drop of $60 \%$ acetic acid. The chromosome preparations were made permanent by freezing the slide in liquid nitrogen, flicking off the coverslip with a razor and then mounting in Euparal.

Photographs of chromosomes were taken using Kodak Technical Pan Film TP120 film using $40 \mathrm{x}$ and $63 \mathrm{x}$ Plan-Apochromat objectives on a Zeiss Universal Microscope. Cytotype identification and chromosome nomenclature follow Procunier et al. (1985). Permanent chromosome preparations and photographs have been deposited in the BM(NH) collections.

As the whole larva is stained in Feulgen, the Feulgen method for polytene chromosome preparation (Dunbar, 1972) has the advantage of concurrently staining the rudimentary gonads as well as the silk glands. The Feulgenstained gonads can easily been seen against the unstained dorsal integument upon removal of the silk glands. The sex of the larva is indicated by the shape of the developing gonads. Female gonads are elongate, whereas males gonads are spherical (Puri, 1925). After sex had been established each larval body was stored in $80 \%$ ethanol for future morphometric analysis.

Isoenzyme electrophoresis - The methods of Herbert \& Beaton (1989) were followed for cellulose acetate electrophoresis (CAE) of total protein homogenates made from adult females of collections 2, 5 and 7 (Table I). In preliminary trials, citric acid $\mathrm{N}$-(3-aminopropyl)-diethylamine (CAEA) tank buffer was used where recommended (Herbert \& Beaton, 1989), but tris-glycine (TG) tank buffer was found to be adequate for all polymorphic enzymes analysed in large samples. Using a short glass rod attached to a slowly-rotating drillbit, most body parts of individual flies were homogenized in $20 \mu \mathrm{l}$ of ice-cold grinding buffer $(0.1 \mathrm{M}$ Tris- $\mathrm{HCl} \mathrm{pH} 8.0,0.5 \%(\mathrm{v} / \mathrm{v})$ Triton $\mathrm{X}-100)$ in the wells of a pre-cooled perspex block placed on ice. The homogenate was divided: NADP (final concentration 10 $\mathrm{mg} / \mathrm{ml}$, necessary for the stability of some enzymes) was added to one lot, and then both lots were stored at $-70^{\circ} \mathrm{C}$. Prior to electrophoresis, $8 \mu$ of each individual homogenate was pipetted into a cooled Helena CAE plateapplicator well. Usually, two applications of homogenate were made to each wetted and blotted CAE plate, enabling at least 8 enzymes ( $=8 \mathrm{CAE}$ plates) to be analysed for each $8 \mu \mathrm{l}$ of homogenate. Each electrophoretic fractionation (or "run") was for $15 \mathrm{~min}$ at 200 volts (100 volts for esterase and in some trials) at room temperature $\left(20^{\circ} \mathrm{C}\right)$ with the tank buffer pre-cooled $\left(4^{\circ} \mathrm{C}\right)$. For each electrophoresis run, an homogenate of a clone of Myzus persicae (Aphididae) was used to provide standard electrophoretic markers for most electromorphs of S. exiguum.

Eighteen enzyme systems were assayed: alcohol dehydrogenase (ADH, E.C. 1.1.1.1), esterase (EST, E.C. 3.1.1.1; substrate alphanaphthyl acetate), glucose-6-dehydrogenase (G6PDH, E.C. 1.1.1.49), glutamate oxaloacetate transferase (GOT, E.C. 2.6.1.1), glycerol3-phosphate dehydrogenase (GPDH, E.C. 1.1.1.8), hexokinase (HEX, E.C. 2.7.1.1), isocitrate dehydrogenase (IDH, E.C. 1.1.1.42), lactate dehydrogenase (LDH, E.C. 1.1.1.27), malate dehydrogenase (MDH, E.C. 1.1.1.37), maleic enzyme (ME, E.C. 1.1.1.40), mannose phosphate isomerase (MP1, E.C. 5.3.1.8), peptidase (PEP, E.C. 3.4.11 or 3.4.13; substrate L-phenylalanine-L-proline), phosphoglucomutase (PGM, E.C. 2.7.5.1), 6-phosphogluconate dehydrogenase (6PGDH, E.C. 1.1.1.44), phosphoglucose isomerase (PGI, E.C. 5.3.1.9), superoxide dismutase (SOD, E.C. 1.15.1.1), trehalase (TRE, E.C. 3.2.1.28) and xanthine dehydrogenase (XDH, E.C. 1.2.1.37). The cellulose acetate plates of all electrophoretic runs have been deposited in the BM (NH) collection.

\section{RESULTS}

Distribution of cytotypes in Ecuador - The data from Procunier et al. (1985) (collection $1)$, the $\mathrm{BM}(\mathrm{NH})$ chromosome slide collection (collection 4) and the new collections (collections 2, 3, 5, 6 and 7) enable us to update the distribution records of $S$. exiguum in Ecuador (Fig. 1).

Cytology - The cytological results are summarized in Table I. S. exiguum possesses the 
typical simuliid complement of three polytene chromosomes $(2 n=6)$. All five larvae (two males, three females) identified from the Rio Tanti (site ERT, see Fig. 1) were of the Cayapa cytotype, which possesses the arbitrarily nominated chromosomal "standard" sequence. The Cayapa cytotype was previously reported to be monomorphic (Procunier et al., 1985). However, in the present study a new inversion, involving the double bubble, designated IIS-B (Fig. 2) was found (frequency of inverted sequence $(f)=0.1$; in one female).

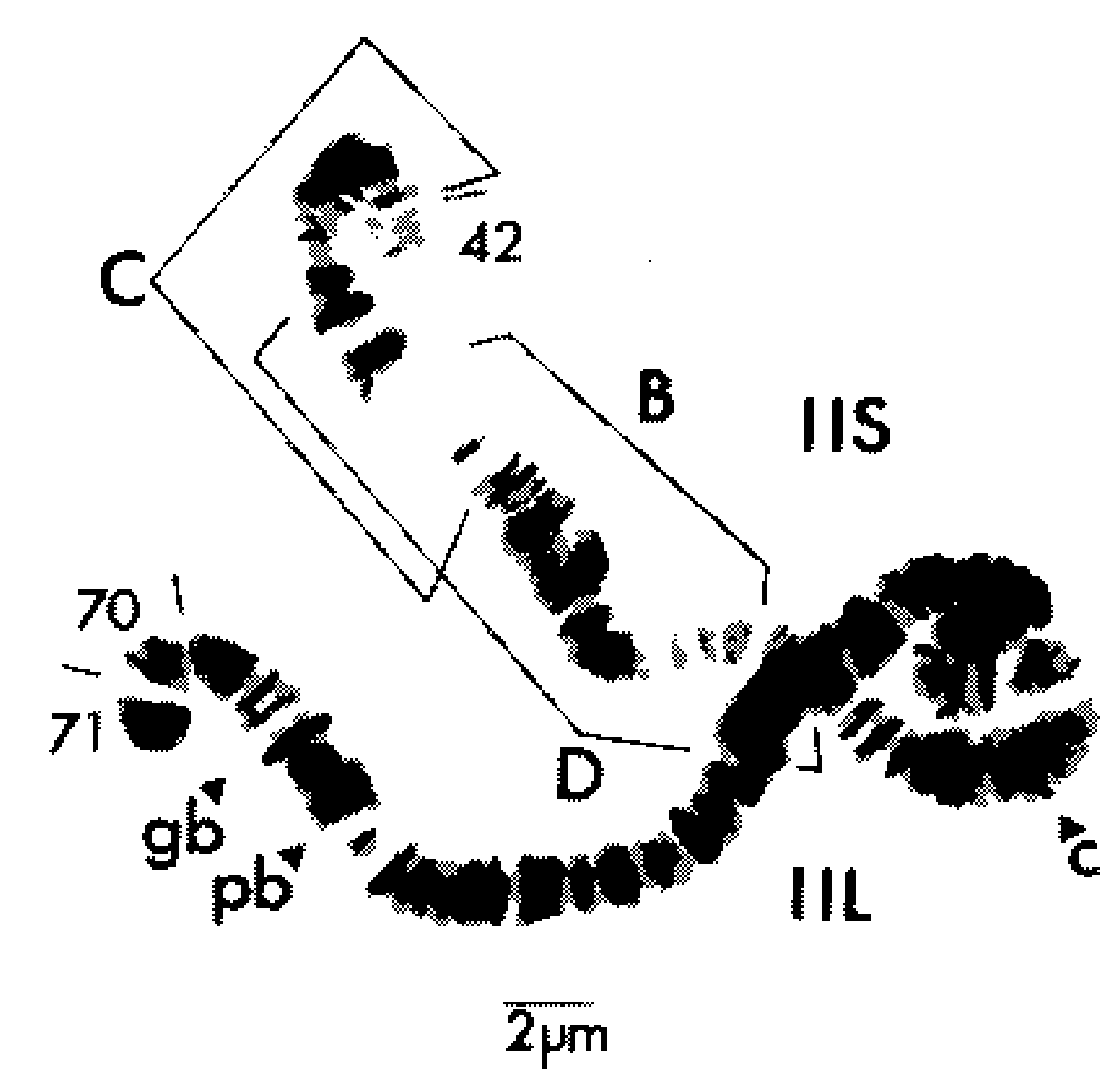

Fig. 2: polymorphic inversions in chromosome Il of Simulium exiguum. The breakpoints of inversions IIS$B$, IIS-C and IIS-D are shown on the standard sequence of the Cayapa cytotype (after Fig. 1. Procunier et al., 1985). Abbreviations as in Fig. 3.

Larvae from the Rio Bucay (EB) were all identified as belonging to the Bucay cytotype. This cytotype differs from the standard sequence of the Cayapa cytotype by two tandemly-arranged fixed inversions: IIL-5 and IIL-6 (IIL-5 + 6; Fig. 3; Table I). The three collections (numbers 1,2 and 3; Table I) made at this site over eight years show that EB remained a pure locality containing only the Bucay cytotype. The Bucay cytotype was originally described as being monomorphic (Procunier et al., 1985; collection 1). While this was so in 1989 (collection 2), a number of polymorphic inversions were found in 1991 (collection 3): IIS-C ( $\mathrm{f}=0.017$; in one male), IIS-D ( $f=0.05$; in one male and one female) (Fig. 2) and IIIL-A ( $\mathrm{f}=0.017$; in one male; Fig. 3).

In 1984, both the Bucay and Cayapa cytotypes were identified from the Rio Pilaló (site EQ, collection 4). Unfortunately, we have no records of inversion frequencies or of heterozygotes for inversions $\mathrm{IIL}-5+6$ in this sample, and the chromosome banding pattern is no longer readable. Only one cytotype (Quevedo) was identified from EQ in 1989 (collection 5) and 1991 (collection 6) (Fig. 4). This cytotype possesses the same fixed inversions as the Bucay cytotype (IIL-5 +6 ), but additionally has a sex-linked inversion. The $\mathrm{X}$-linked inversion, designated IIS-A (Figs 3 , 4), occurs in the short arm of chromosome II, and involves the double-bubble. A number of autosomal polymorphic inversions were also present. IILL-A, which is shared with the Bucay cytotype, was found in 1989 (collection $5 ; \mathrm{f}=$ 0.038 ; in one male) but not in 1991 (collection 6). In 1991 (collection 6) the autosomal polymorphisms designated IL-B ( $\mathrm{f}=0.024$; in one fcmale), IIIL-B ( $f=0.024$; in one female) and IIIL-C ( $f=0.024$; in one male; Fig. 3 ) were present in the EQ population.

Whilst not giving a chromosomal description of the Quevedo cytotype, Procunicr (1989: 561) states that "the Quevedo cytoform differs from the Bucay sibling by having distinct sex chromosomes". The collections made at Rio Pilalo (EQ) are from the same site in which the Quevedo cytotype was originally found (Shelley \& Procunier, unpublished observations). Together, these facts indicate that the IIS-A inversion is the rearrangement referred to by Procunier (1989) as being the sex chromosome difference between the Bucay and Quevedo cytotypes. The Bucay cytotype possesses undifferentiated sex chromosomes $\left(\mathrm{X}_{0} \mathrm{X}_{0}\right.$ females and $X_{0} Y_{0}$ males). All Quevedo cytotype females were found to be $X_{1} X_{1}(n=$ $21)$ and the males were all $X_{1} Y_{0}(n=13)$. All the known cytological differences found between the Bucay and Quevedo cytotypes are summarized in Fig. 5.

In 1989 and 1991 the Bucay and Quevedo cytotypes were found in pure populations at the EB and EQ sites, respectively (Table I). It remains possible that other cytotypes might have occurred but that they were not found because of small sample sizes. The maximum frequency another cytotype could have attained can be calculated using the binomial probability. In 1991, at the Rio Bucay (EB) site the maximum frequency is $9.5 \%$, while for the Río Pilaló (EQ) site it is $13.3 \%$; if another cytotype had been present at a higher frequency there would have been a $95 \%$ probability of detecting it (see Post \& Millest, 1991).

Isoenzymes - Only adult females from col- 

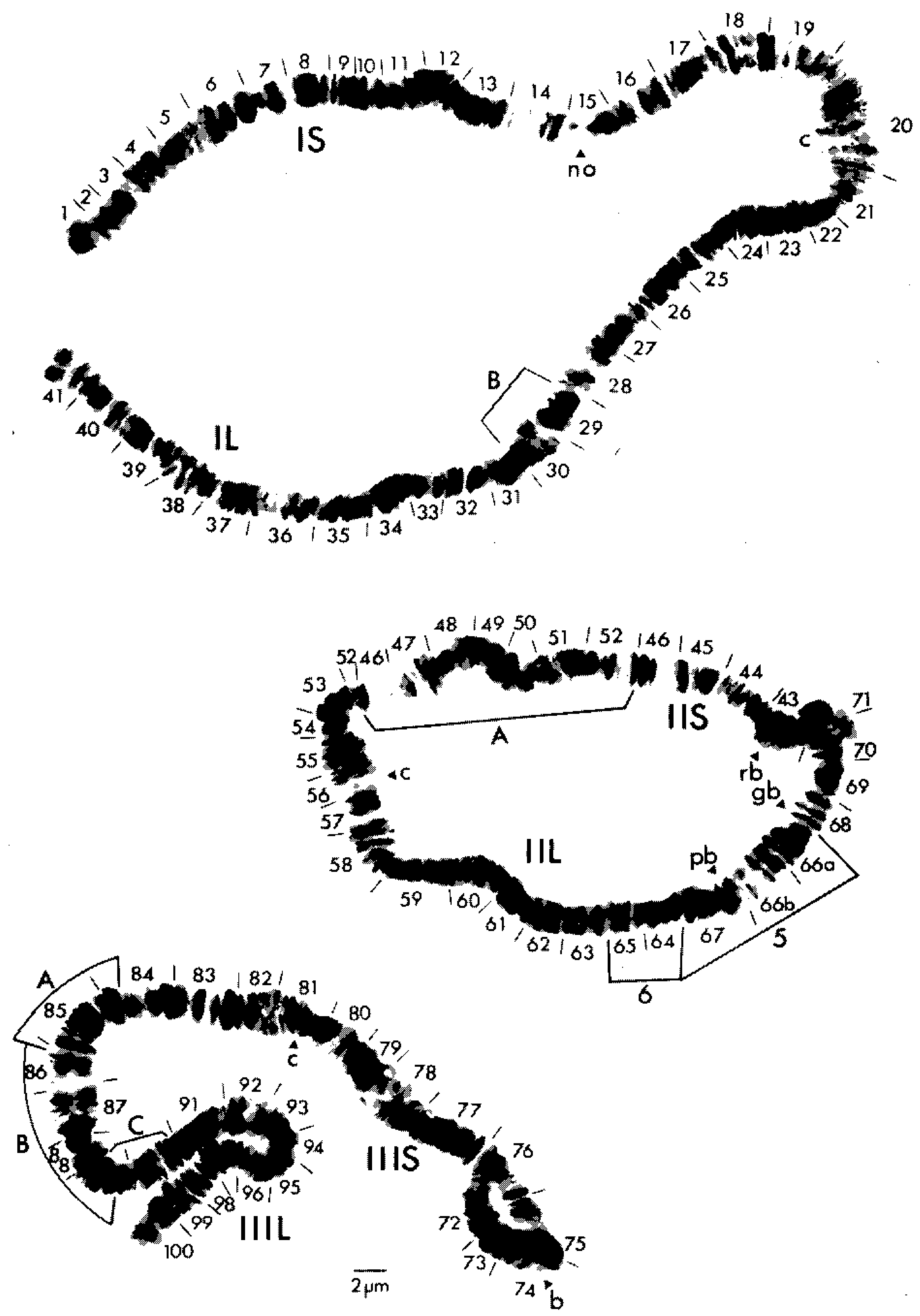

Fig. 3: polytene chromosomes of a Quevedo female, collected from the Rio Soloma (17.9.91). Inversions IIS-A and IIL- $5+6$ are present in the homozygous state. The breakpoints of inversions IL-B, IIIL-A, IIIL-B and IIL-C are shown. Sections 42, 70 and $7 \mathrm{I}$ can be seen more clearly in Fig. 2. I, II, Ill = chromosome number; $\mathrm{S}(\mathrm{L})=$ short (long) arm of chromosome: $\mathrm{c}=$ centromere; no = nucleolar organiser; $\mathrm{rb} \cdot$. Ring of Balbiani; $\mathrm{pb}=$ parabalbiani; gb $=$ grey band; $b=$ bubble.

lections 2, 5 and 7 (Table I) were tested (in the first half of 1991).

Attempts were made to reveal 18 different enzymes, using at least four adults of each sample. Each $20 \mu \mathrm{l}$ of homogenate from an individual fly provided for at least 20 electrophoretic runs (i.e. all 18 enzymes could be analysed from one fly).

Four enzymes were easily detected and it was routinely possible to interpret the poly- 


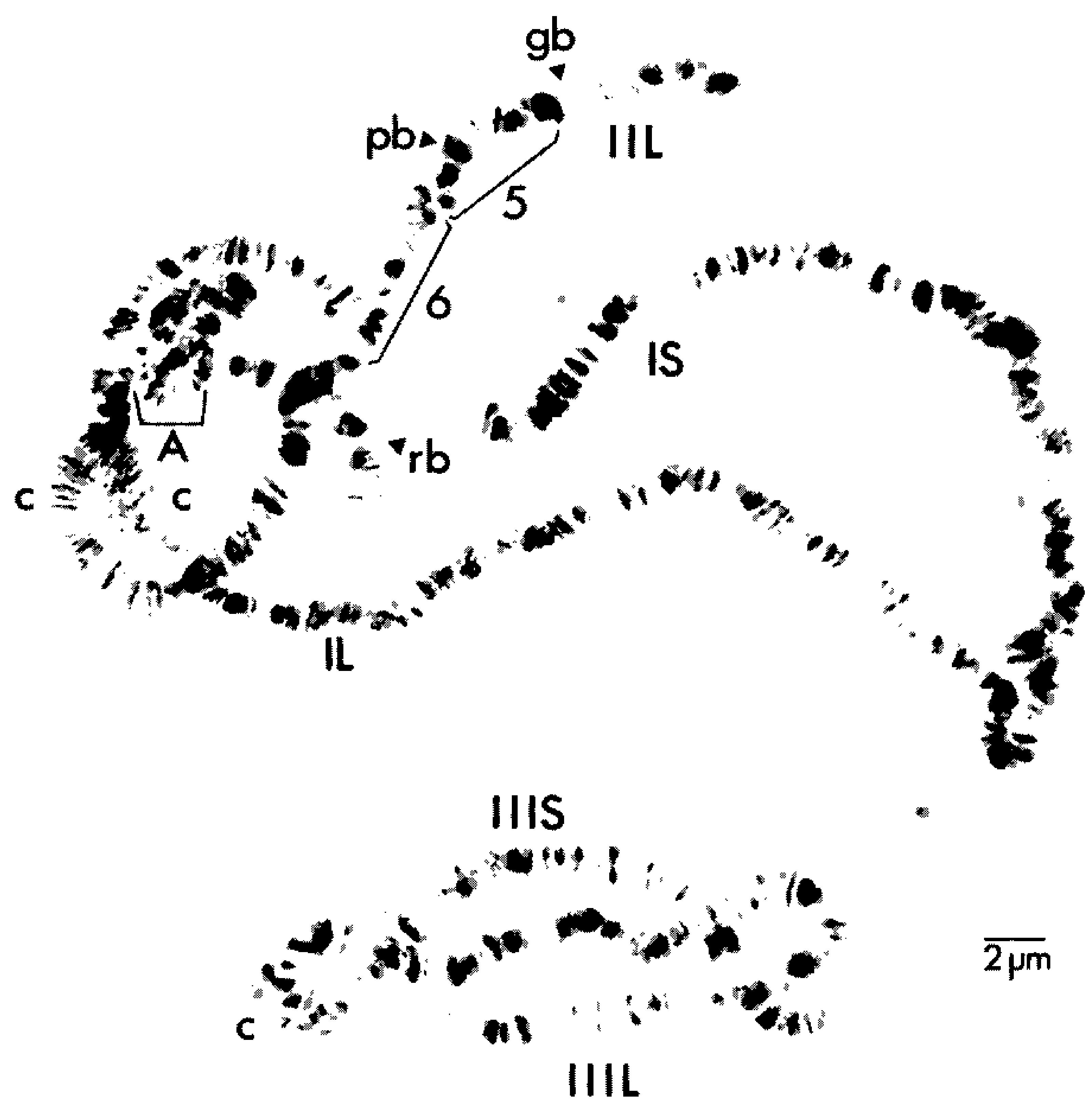

Fig. 4: polytene chromosomes of a Quevedo male, collected from the Rio Pilalo (24.9.91). The fixed inversions IIL$5+6$ and the X-linked inversion IIS-A are present. Abbreviations as in Fig. 3.

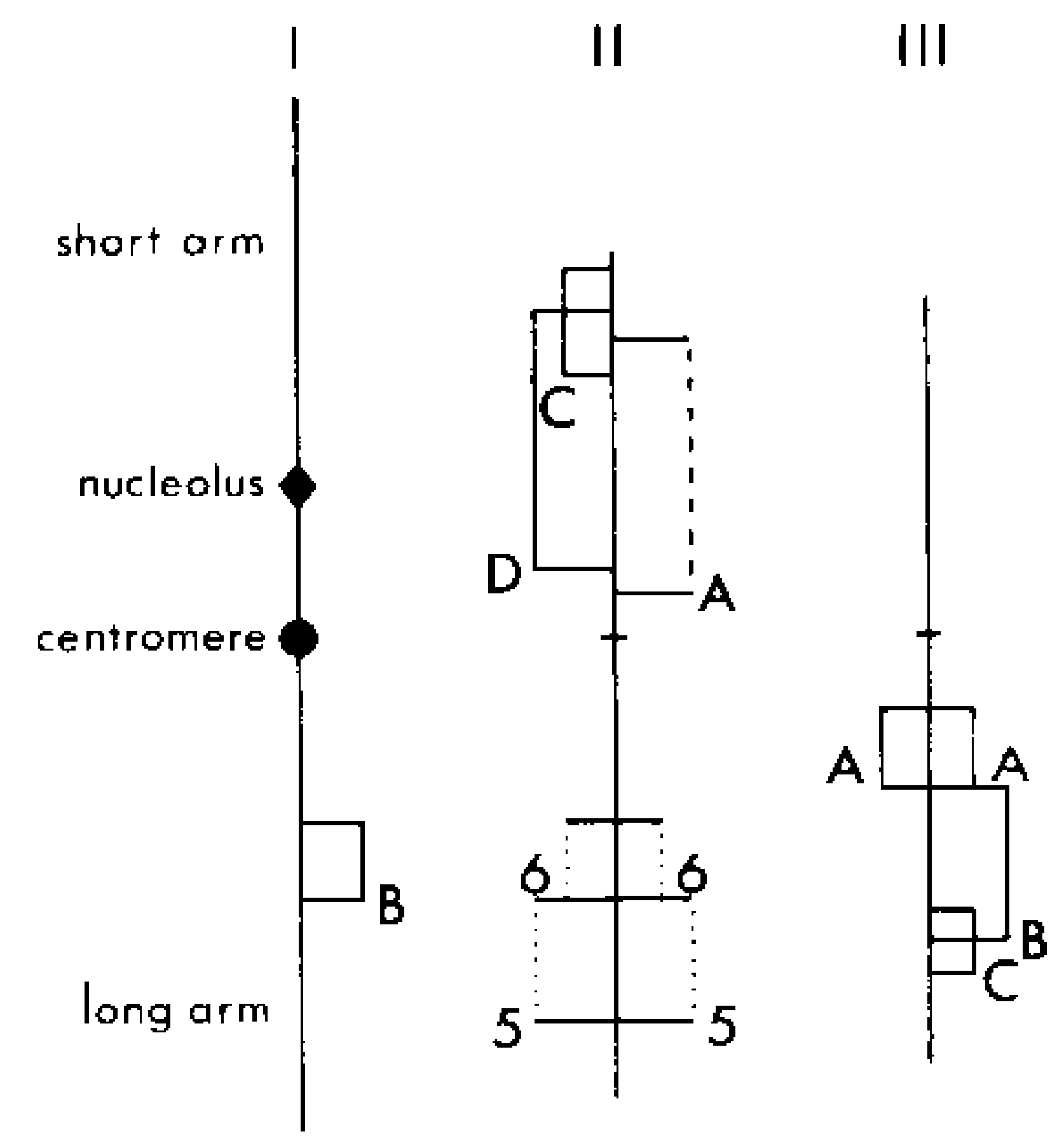

Fig. 5: idiogram of the Bucay and Quevedo cytotypes compared with the standard sequence of the Cayapa cytotype. Inversions shown on the left and right occur in the Bucay and Quevedo cytotypes, respectively. Dotted, dashed and solid lines mark the fixed, sex and polymorphic inversions. morphism shown, i.e. two or more electromorphs (= isoenzymes) were regularly revealed and their relative mobilities could be assessed accurately: PGM, PGI, IDH (faster migrating supernatant form) and $\mathrm{ME}$.

Eight enzymes were routinely revealed, but polymorphism was either absent (3) or sometimes unreadable (e.g. electromorphs of TRE migrated for similar, overlapping distances and could not always be distinguished with certainty): GPDH, HEX, MDH, MPI, PEP, 6PGDH, TRE and XDH.

Six other enzymes could not be detected in S. exiguum: ADH, EST, G6PDH, GOT, LDH and SOD.

Diagnostic isoenzymes - No electromorphs were found that identified all or most adult females of each collection. Only one resolved electromorph was found to be restricted to one 


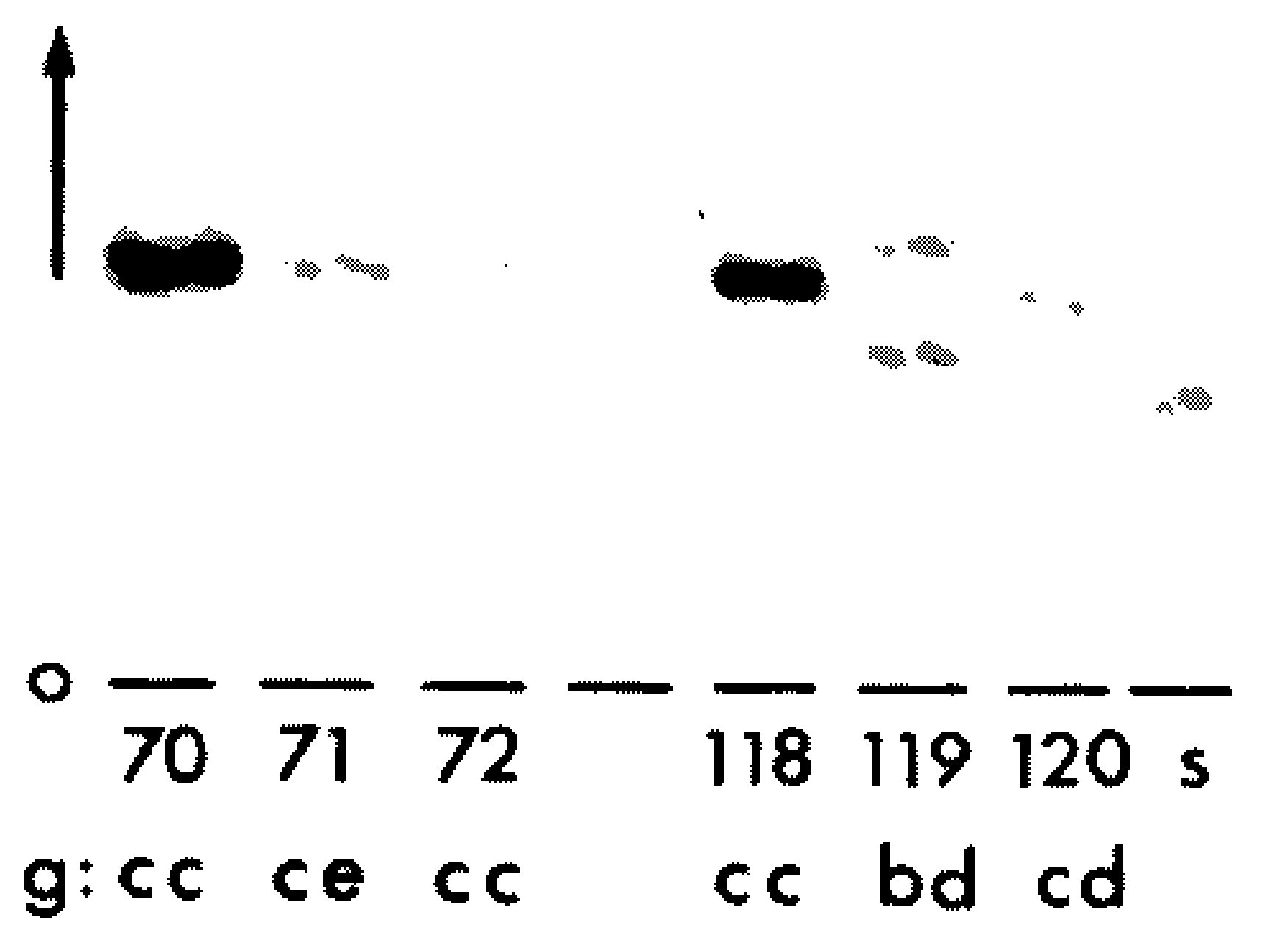

Fig. 6: PGM electromorphs (anodic) of individual females of Simulium exigurm. Specimen nos. 70-72: Bucay cytotype from collection 2;118-120: Quevedo cytotype from collection $5 . \mathrm{g}=$ genotype; $\mathrm{s}=$ standard clone of $M$. persicae; $0=$ origin: $-=5 \mathrm{~mm} ; \mathbf{t}=$ anodic direction of migration.

TABLE II

Frequencies of alleles and genotypes of PGM

(a) Frequencies of alleles of PGM

\begin{tabular}{cccc}
$\begin{array}{c}\text { Collection no. } \\
\downarrow \text { Allele }\end{array}$ & 2 & 5 & 7 \\
\hline a & 0.0556 & 0.0000 & 0.0000 \\
b & 0.0833 & .0 .1316 & 0.0625 \\
c & 0.7685 & 0.5088 & 0.4167 \\
d & 0.0741 & 0.3333 & 0.5104 \\
e & 0.0185 & 0.0263 & 0.0104 \\
\hline
\end{tabular}

(b) Observed (and expected) frequencies of genotypes of PGM

\begin{tabular}{crrrrrr}
\hline $\begin{array}{c}\text { Collection no. } \rightarrow \\
\downarrow \text { Genotype }\end{array}$ & \multicolumn{1}{l}{2} & & & & & 7 \\
\hline aa & 0 & $(0.140)$ & 0 & $(0.000)$ & 0 & $(0.000)$ \\
ab & 0 & $(0.505)$ & 0 & $(0.000)$ & 0 & $(0.000)$ \\
ac & 4 & $(4.658)$ & 0 & $(0.000)$ & 0 & $(0.000)$ \\
ad & 1 & $(0.449)$ & 0 & $(0.000)$ & 0 & $(0.000)$ \\
ae & 1 & $(0.112)$ & 0 & $(0.000)$ & 0 & $(0.000)$ \\
bb & 1 & $(0.336)$ & 2 & $(0.930)$ & 0 & $(0.158)$ \\
bc & 5 & $(6.978)$ & 6 & $(7.700)$ & 4 & $(2.527)$ \\
bd & 2 & $(0.673)$ & 4 & $(5.044)$ & 2 & $(3.095)$ \\
be & 0 & $(0.168)$ & 1 & $(0.398)$ & 0 & $(0.063)$ \\
cc & 35 & $(31.802)$ & 19 & $(14.630)$ & 7 & $(8.212)$ \\
cd & 3 & $(6.208)$ & 14 & $(19.504)$ & 21 & $(20.633)$ \\
ce & 1 & $(1.550)$ & 0 & $(1.539)$ & 1 & $(0.420)$ \\
dd & 1 & $(0.262)$ & 9 & $(6.220)$ & 13 & $(12.378)$ \\
de & 0 & $(0.149)$ & 2 & $(1.008)$ & 0 & $(0.515)$ \\
ee & 0 & $(0.009)$ & 0 & $(0.027)$ & 0 & $(0.000)$ \\
\hline Total & & 54 & & 57 & & 48 \\
\hline
\end{tabular}

collection, namely allele a of PGM in 6 out of 54 females from collection 2 (see below).

Allele and genotype frequencies of PGM Only this enzyme displayed polymorphism that differentiated the collections analyzed (other results will be presented elsewhere following further collecting). The different electromorphs of PGM occurred singly or in pairs in individual female flies and in a manner consistent with there being in the populations a total of five co-dominant alleles at a single locus for this dimeric enzyme. Alleles (revealed as isoenzymes) were scored as a-e (with decreasing anodic mobility) and were identified in different electrophoretic runs by reference to a standard electromorph from a clone of $M$. persicae; the presumed diploid genotypes are shown in Fig. 6. The observed frequencies of each allele and genotype are given for each collection in Table II, along with the expected frequencies of each genotype based on Hardy-Weinberg ratios. For many genotypes, the expected frequencies are less than five, and so statistical comparisons (Chi-square) are not accurate (Sokal \& Rohlf, 1981). This is common with such data and therefore, following usual practice, genotypes have been grouped to permit analysis. From Table II it is clear that the major differences between samples lies in the frequencies of alleles $\mathbf{c}$, $\mathbf{d}$ and perhaps $\mathbf{b}$. Consequently, genotypes were grouped so that, for these three alleles, their homozygotes $(\mathbf{b b}$, cc, dd) and most frequent heterozygotes (bc, cd) were not combined, with the constraint that expected frequencies of each genotype had to be greater than five (Table III).

Comparisons of the observed and expected frequencies in Table III showed a highly sig-

TABLE III

Frequencies of grouped genotypes of PGM

\begin{tabular}{crrrrrr}
\hline & \multicolumn{4}{c}{ Observed } & $\begin{array}{c}\text { Expected } \\
\text { (Hardy-Weinberg) }\end{array}$ \\
\cline { 2 - 6 } $\begin{array}{c}\text { Collection no. } \\
\begin{array}{c}\text { Grouped } \\
\downarrow \text { Genotypes }\end{array}\end{array}$ & 2 & 5 & 7 & Combined & Combined \\
\hline bb \&bd & 3 & 6 & 2 & 11 & 10.5 \\
be & 5 & 6 & 4 & 15 & 17.2 \\
cc & 35 & 19 & 7 & 61 & 51.5 \\
cd & 3 & 14 & 21 & 38 & 54.3 \\
dd & 1 & 9 & 13 & 23 & 14.1 \\
"others" & 7 & 3 & 1 & 11 & 11.4 \\
\hline Total & 54 & 5748 & 159 & 159 \\
\hline
\end{tabular}


nificant deviation from Hardy-Weinberg ratios when the samples were combined (Chi-square $=12.6$, probability $(\mathrm{p})<0.05)$ but not for each individual population treated alone (for which $\mathbf{b b}+\mathbf{b d}$ and bc had to be grouped with "others"; Chi-square $<1.8, \mathrm{p}>0.05$ ).

Further, when combining samples in pairs $(2+5,2+7,5+7)$, a significant difference from Hardy-Weinberg ratios was found (Chisquare $>12.0, p<0.01$ ) only when collection 2 (which was a pure Bucay cytotype locality) was included. These results indicate that collection 2 was from a population that was not freely interbreeding with the others (i.e. not panmictic), although the null hypothesis of panmixis was not rejected for each of the populations treated individually.

\section{DISCUSSION}

The Quevedo cytotype of $S$. exiguum has now been described from its "type locality", and is clearly closely-related to the Bucay cytotype as they share the same fixed inversions (IIL-5 + 6). It differs from the Bucay cytotype $\left(\mathrm{X}_{0} \mathrm{X}_{0}\right.$ females, $\mathrm{X}_{0} \mathrm{Y}_{0}$ males $)$ in having a differentiated $X$ chromosome $\left(X_{1} X_{1}\right.$ females, $X_{1} X_{0}$ males), indicated by inversion IIS$A$, which occurred in all larvae examined from collections 5 and 6 . An analogous situation is found with two members of the $S$. damnosum complex in West Africa. Simulium sirbanum and $S$. sudanense share the same fixed inversions (relative to the standard) but were considered different cytospecies as the inversion IS-3 is linked with the most frequent X chromosome in $S$. sudanense but with the most frequent Y chromosome in S. sirbanum (Vajime \& Dunbar, 1975). However, analysis of new samples suggests that the only difference between the two putative cytospecies is that $S$. sudanense possesses a differentiated $\mathrm{X}$ chromosome, indicated by the inversion IS-3 (Vajime, 1989). If there had been two cytospecies, only four chromosomal classes of individuals should have occurred in the sympatric populations: females and males homozygous for the standard sequence (IS-st/st; i.e. $\mathrm{X}_{0} \mathrm{X}_{0}$ and $\mathrm{X}_{0} \mathrm{Y}_{0}$ ) representing $S$. sirbanum; and females homozygous for the inverted sequence (IS-3/3; i.e. $X_{1} X_{1}$ ) and males heterozygous for IS-3 (IS-st $/ 3$; i.e. $X_{1} Y_{0}$ ) representing $S$. sudanense. Vajime (1989) found, though, that females heterozygous (IS-st/3) and males homozygous (IS-3/3) for the inverted sequence occurred in sympatric populations. The pres- ence of these other chromosomal classes suggests that reproductive isolation is not complete and, therefore, two cytospecies are not present. However, the occurrence of these other chromosomal classes is at such low frequencies as to imply that the population is not panmictic, but that two chromosomal forms of one species, $S$. sirbanum, do occur and shown a high, but incomplete, degree of reproductive isolation.

The Bucay and Quevedo cytotypes have not yet been analyzed in sympatric populations. Therefore, the occurrence of females heterozygous for IIS-A (i.e. $\mathrm{X}_{0} \mathrm{X}_{1}$ ) and the degree of reproductive isolation between these cytotypes is unknown. Consequently, at present, Quevedo and Bucay must be considered to be chromosomal forms of one cytotype, Bucay.

The isoenzyme characterization indicated that the Bucay and Quevedo cytotypes are not differentiated to the extent shown by closelyrelated simuliid morphospecies: the allelic similarity was $95 \%$ over 8 loci ( 20 "major" alleles, i.e. each with overall frequency of $>1 \%$ ) or $100 \%$ over the same 8 loci $(9$ "major" alleles, each with frequency $>50 \%$ in any one population), whereas it was $64 \%$ between $S$. horacioi and $S$. metallicum (or $S$. ochraceum) over 8 loci (14 "major" alleles, i.e. each with frequency of $>50 \%$ in any one population) (Agatsuma et al., 1986). Moreover, unlike the latter three species, no major alleles were unique to one or other population- allele a of PGM was restricted to collection 2 (which was cytologically pure for the Bucay cytotype) but at a frequency too low for detailed genetic analysis or diagnostic purposes (Table II).

The differences in PGM genotype frequencies between the two samples believed to be pure for the Bucay and Quevedo cytotypes (samples 2 and 5 , respectively) are consistent with those between regionally (and temporarily) isolated populations of one biological species. Besides being separated by distance, the three populations were collected in different drainage systems and at different altitudes (increasing south to north), and these are just some of the factors that might isolate the cytotypes.

The principal limitation of our investigations so far has been the absence of adequate material from sympatric populations, in which a deficiency of chromosomal or isoenzyme heterozygotes would indicate specific status. The 
same limitation means that the "taxonomic" status of the Bucay cytotype as a "sibling species" (Procunier, 1989) shoud not be assumed as most of the specimens characterized so far have been collected from one locality (Río Bucay at Naranjal). For example, a possible scenario is that inversions IIL-5 +6 are polymorphic, rather than fixed, and are found at $100 \%$ frequency in the Bucay and Quevedo populations as these represent one end of inversion-frequency clines. This is reflected by the possible clines in frequencies of PGM alleles $\mathbf{c}$ and $\mathbf{d}$, with the Bucay population more markedly differentiated at the southern end (Table II).

The discovery in the $\mathrm{BM}(\mathrm{NH})$ collections of Bucay and Cayapa cytotypes collected at the same time from the Quevedo site (EQ; Table I) clearly shows that the distributions of some of the cytotypes of $S$. exiguum do overlap in some areas. Further collections are being made from putatively allopatric and sympatric populations, so that we can investigate more fully the genetic relationships of these cytotypes.

\section{ACKNOWLEDGEMENTS}

To J. R. Dagley, I. Mera and A. Villavicencio for help during collecting in Ecuador. To J. Sawyer-Wallbanks for the Rio Tanti collection, T. M. Howard and J. Testa for technical support, P. V. York for photomicrography, L. M. Huddleston for help in preparing the manuscript and T. M. Howard for drawing Fig. 1.

\section{REFERENCES}

AGATSUMA, T.; UEMOTO, K. \& OCHOA, J. O., 1986. Biochemical genetics of blackfly isozymes I. Isozyme variation among three species, Simulium ochraceum, $S$. metallicum and $S$. horacioi from Guatemala. Jpn. J. Sanit. Zool., 37: 1-9.

DUKE, B. O. L., 1970. Onchocerca-Simulium complexes VI. Experimental studies on the transmission of Venezuelan and West African strains of Onchocerca volvulus by Simulium metallicum and $S$. exiguum in Venezuela. Ann. Trop. Med. Parasitol., 64: 421. 431.

DUNBAR, R. W., 1972. Polytene chromosome preparations from tropical Simuliidae. (WHO Mimeographed Document): WHO/ONCHO/Geneva 72.95 : 1-16.
HERBERT, P. D. N. \& BEATON, M. J., 1989. Methodologies for allozyme analysis using cellulose acetate electrophoresis - A Practical Handbook. Helena Laboratories, Windsor, Ontario. p. 1-31.

POST, R. J. \& MILLEST, A. L., 1991. Sample size in parasitological and vector surveys. Parasitology Today, 7: 141.

PROCUNIER, W. S., 1989. Cytological approaches to simuliid biosystematics in relation to the epidemiology and control of human onchocerciasis. Genome, 32: 559-569.

PROCUNIER, W. S.; SHELLEY, A. J. \& ARZUBE, M., 1985. Sibling species of Simulium exiguum: the primary vector of onchocerciasis in Ecuador. Rev. Ecuat. Hig. Med. Trop., 35: 49-59.

PURI, I. M., 1925. On the life history and structure of the early stages of Simuliidae (Diptera, Nematocera). Parasitology, 17: 295-334.

SHELLEY, A. J., 1988. Biosystematics and medical importance of the Simulium amazonicum group and the $S$. exiguum complex in Latin America, p. 203 220. In M. W. Service Biosystematics of Haematophagous Insects. Systematics Association Special Volume No. 37. Clarendon Press, Oxford.

SHELlEY, A. J. \& ARZUBE, M., 1985. Studies on the biology of the Simuliidae (Diptera) at the Santiago onchocerciasis focus in Ecuador, with special reference to the vectors and disease transmission. Trans. R. Soc. Trop. Med. Hyg., 79: 328-338.

SHELLEY, A. J.; ARZUBE, M. \& COUCH, C. A., 1989. The Simuliidae (Diptera) of the Santiago onchocerciasis focus of Ecuador. Bull. Br. Mus. nat. Hist. (Ent.)., 58: 79-130.

SHELLEY , A. J.; PROCUNIER, W. S. \& ARZUBE, M., 1985. Direct vector incrimination of Simulium exiguum Cayapa form, as a vector of Onchocerca volvulus in Ecuador. Trans. R. Soc. Trop. Med. Hyg., 80: 845 .

SHELLEY, A. J.; PROCUNIER, W. S. \& ARZUBE, M., 1990. Desarrollo de la Onchocerca volvulus en dos citospecies de Simulium exiguum complex (Diptera: Simuliidae) en el Ecuador. Rev. Ecuat. Hyg. Med. Trop., 39: 9-23.

SOKAL, R. R. \& ROHLF, F. J., 1981. Biometry. Freeman. San Francisco. p. 859.

TIDWELL, M. A.; TIDWELL, M. A.; MUNOS DE HOYOS, P. \& CORREDOR, A., 1980. Simulium exiguum, the vector of Onchocerca volvulus on the Rio Micay, Colombia. Am. J. Trop. Med. Hyg., 29: 77-381.

VAJIME, C. G., 1989. Cytotaxonomy of Sirba form populations of the Simulium damnosum complex in West Africa: amendments to sex chromosomes and sibling status. Trop. Med. Parasit., 40: 464-467.

VAJIME, C. G. \& DUNBAR, R. W., 1975. Chromosomal identification of eight species of the subgenus Edwardsellum near and including Simulium (Edwardsellum) damnosum Theobald (Diptera: Simuljidae). Tropenmend. Parasit., 26: $111-138$. 